\title{
Terminal Sliding Mode Control Using Adaptive Fuzzy-Neural Observer
}

\author{
Dezhi Xu, Bin Jiang, Moshu Qian, and Jing Zhao \\ College of Automation Engineering, Nanjing University of Aeronautics and Astronautics, Nanjing 210016, China \\ Correspondence should be addressed to Bin Jiang; ebjiang@yahoo.com
}

Received 31 October 2012; Revised 27 December 2012; Accepted 27 December 2012

Academic Editor: Peng Shi

Copyright ( 2013 Dezhi Xu et al. This is an open access article distributed under the Creative Commons Attribution License, which permits unrestricted use, distribution, and reproduction in any medium, provided the original work is properly cited.

\begin{abstract}
We propose a terminal sliding mode control (SMC) law based on adaptive fuzzy-neural observer for nonaffine nonlinear uncertain system. First, a novel nonaffine nonlinear approximation algorithm is proposed for observer and controller design. Then, an adaptive fuzzy-neural observer is introduced to identify the simplified model and resolve the problem of the unavailability of the state variables. Moreover, based on the information of the adaptive observer, the terminal SMC law is designed. The Lyapunov synthesis approach is used to guarantee a global uniform ultimate boundedness property of the state estimation error and the asymptotic output tracking of the closed-loop control systems in spite of unknown uncertainties/disturbances, as well as all the other signals in the closed-loop system. Finally, using the designed terminal sliding mode controller, the simulation results on the dynamic model demonstrate the effectiveness of the proposed new control techniques.
\end{abstract}

\section{Introduction}

Sliding mode control (SMC) is known to be a robust control scheme applicable for controlling uncertain systems. Great robustness is provided against various categories of uncertainties such as external disturbances and measurement errors [1-7]. It is also straightforward to execute the resulting algorithms. The dynamics performance of a SMC system is affected by the suggested sliding manifolds upon which the control structure is switched. The most normally used sliding manifolds are linear hyperplanes. Such hyperplanes ensure the asymptotic stability of the sliding mode. Nonlinear switching manifolds may give increase to a better control performance, provided the nonlinearity is added purposefully. The terminal sliding mode concept has been suggested to focus the finite time control [8]. However, SMC techniques cannot provide satisfactory results when suffering poorly modeling unless the designers know the bounds of uncertainty.

Since fuzzy logic systems (FLSs) and neural networks (NNs) are universal function approximators [9-16], the adaptive control methods of nonlinear systems that integrate the universal function approximators have developed quickly $[2,13,17]$. The main improvement of using universal function approximators is to eliminate the linear-in-the-parameters form on unknown nonlinearities of the controlled pant. Thus, the class of nonlinear systems to which the adaptive control technique can be employed is improved by using universal function approximators. Another benefit is that we can use conventional and advanced adaptive techniques with a novel robust action which compensates the errors.

In fact, most of the works in the fuzzy/neural control are dedicated to the control problem for the affine nonlinear systems, that is, systems characterized by inputs appearing linearly in the system input-output equation. Few results are accessible for nonaffine nonlinear systems in which the control input occurs in a nonlinear fashion. In this paper, a novel dynamic model approximation method is first proposed to approximate the nonaffine nonlinear dynamics, which is a solution that bridges the gap between affine and nonaffine control systems. Then we combine the FLSs and NNs, and adaptive techniques proposed an adaptive fuzzyneural observer for nonaffine nonlinear systems. Because in many control problems, state variables may be partly unavailable. So an adaptive observer is designed, and it entails simultaneous estimation of parameters and unknown state variables. The update laws of fuzzy-neural network (FNN) parameters provide the Lyapunov stability for the closed-loop 


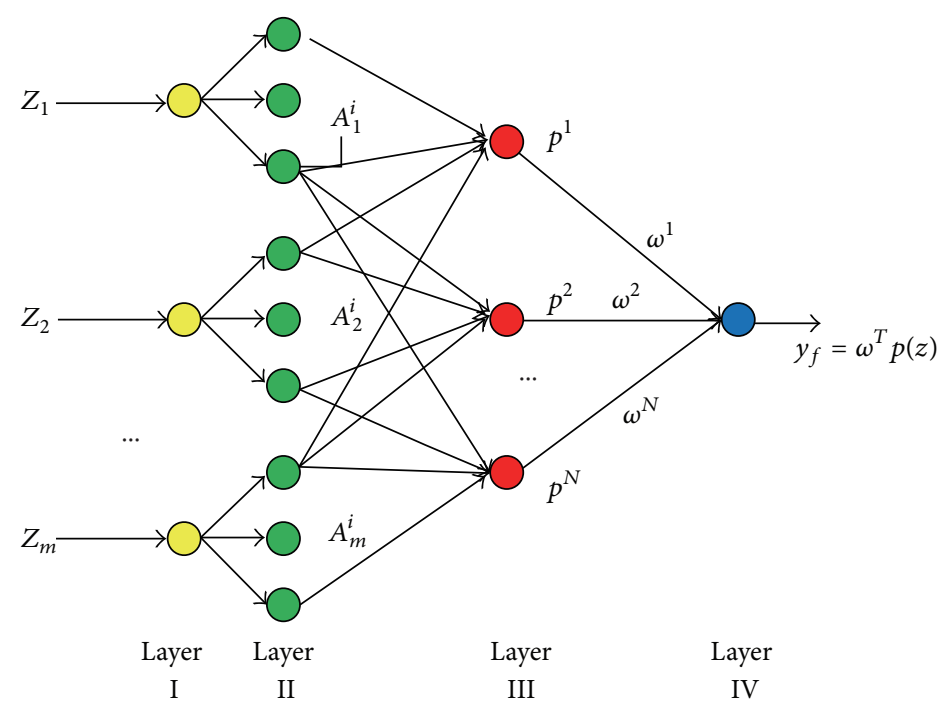

FIGURE 1: Functional link of a fuzzy-neural network structure.

system and guarantee that all signals involved are globally uniformly ultimately bounded. Using terminal SMC, based on the adaptive FNN observer, the robust tracking controller is designed.

The rest of this paper is organized as follows. First, a brief descriptions of the fuzzy-neural network system. Main results include a novel dynamic model approximation technique, adaptive fuzzy-neural observer, and an SMC control algorithm which are proposed in Section 3. In Section 4, simulation results are presented to show the effectiveness of the proposed technique. Finally, some conclusions are made at the end of this paper.

\section{Background}

2.1. Fuzzy-Neural Network for Approximation. Figure 1 depicts a functional link FNN which consists of fuzzy logic and neural network. The FLS can be divided into two parts: some fuzzy IF-THEN rules and a fuzzy inference engine. The fuzzy inference engine uses the fuzzy IF-THEN rules to perform a mapping from an input linguistic vector $z=\left[z_{1}, \ldots, z_{m}\right]^{T} \in \mathfrak{R}^{m}$ to a scalar output variable $y_{f} \in \mathfrak{R}$. The $i$ th fuzzy IF-THEN rule can be characterized by the following form [16]:

$$
\begin{aligned}
& \text { IF } z_{1} \text { is } A_{1}^{i} \text { and } \cdots \text { and } z_{m} \text { is } A_{m}^{i} \\
& \text { THEN } y_{f} \text { is } B^{i} \quad(i=1, \ldots, N) \text {, }
\end{aligned}
$$

where $A_{j}^{i}, B^{i}$ are fuzzy sets. By using product inference, center-average and singleton fuzzifier, $N$ is the total number of rules. Then the output of the FNN can be expressed as

$$
y_{f}=\frac{\sum_{i=1}^{N} \omega^{i}\left[\prod_{j}^{m} \mu_{A_{j}^{i}}\left(z_{j}\right)\right]}{\sum_{i=1}^{N}\left[\prod_{j}^{m} \mu_{A_{j}^{i}}\left(z_{j}\right)\right]}=W^{T} P(z),
$$

where $\mu_{A_{j}^{i}}\left(z_{j}\right)$ is the membership function value of the fuzzy variable, $\omega^{i}$ is the point at which $\mu_{B^{i}}\left(\omega^{i}\right)=1$, and $W=\left[\omega^{1}, \omega^{2}, \ldots, \omega^{N}\right]$ is an adjustable parameter vector. We assume that an upper limit $\|\varepsilon(z)\| \leq \varepsilon_{M}$ of the functional reconstruction error is known. $P=\left[p^{1}, p^{2}, \ldots, p^{N}\right]$ is a fuzzy basis vector, where $p^{i}$ is defined as

$$
p^{i}(z)=\frac{\prod_{j}^{m} \mu_{A_{j}^{i}}\left(z_{j}\right)}{\sum_{i=1}^{N}\left[\prod_{j}^{m} \mu_{A_{j}^{i}}\left(z_{j}\right)\right]} .
$$

The truth value $p^{i}$ (layer III) of the antecedent part of the $i$ th implication is calculated by (3). Among the commonly used defuzzification strategies, the output (layer IV) of the FNN is expressed as (2). The fuzzy logic approximator based on the neural network can be established. The approximator has four layers. At layer I, nodes, which are input ones, stand for the input linguistic variables. At layer II, nodes represent the values of the membership function value. At layer III, nodes are the values of the fuzzy basis vector. Each node of layer III performs a fuzzy rule. The links between layer III and layer IV are full connected by the weighting vector. $\omega$, that is, the adjusted parameters. At layer IV, the output stands for the value of $y_{f}$.

\section{Main Results}

Consider the nonaffine nonlinear system represented in the following normal form:

$$
\begin{gathered}
\dot{x}_{i}=x_{i+1}, \quad i=1,2, \ldots, n-1, \\
\dot{x}_{n}=f(x, u)+d(t), \\
y=x_{1},
\end{gathered}
$$


or equivalently in observer canonical form given by

$$
\begin{gathered}
\dot{x}=A x+b[f(x, u)+d(t)], \\
y=c^{T} x,
\end{gathered}
$$

where

$$
A=\left[\begin{array}{ccccc}
0 & 1 & 0 & \cdots & 0 \\
0 & 0 & 1 & \cdots & 0 \\
& & & \cdots & \\
0 & 0 & 0 & \cdots & 1 \\
0 & 0 & 0 & \cdots & 0
\end{array}\right], \quad b=\left[\begin{array}{c}
0 \\
0 \\
\vdots \\
0 \\
1
\end{array}\right], \quad c=\left[\begin{array}{c}
1 \\
0 \\
\vdots \\
0 \\
0
\end{array}\right]
$$

and $x=\left[x_{1}, \ldots, x_{n}\right]^{T} \in \mathfrak{R}^{n}$ is the state vector of the system in the normal form which is assumed to be available for measurement, $u \in \mathfrak{R}$ is the control input assumed to have an upper bound $\bar{u}$ (i.e., $|u| \leq \bar{u}$ ), $y \in \mathfrak{R}$ is the system output, and $f(x, u)$ is an unknown smooth nonlinear function. $d(t)$ denotes the external disturbance.

Observer-based model identification methods have been proposed in affine nonlinear systems in $[18,19]$. However, using such observers to identify for nonaffine nonlinear systems is not an easy thing. And in many practical situations, state variables are often unavailable in nonlinear systems. Thus, observer-based adaptive fuzzy control is required for such complicated applications. In this section, in order to design robust tracking controller for unknown nonaffine nonlinear uncertain systems, a new model-free control design method is firstly proposed based on sliding mode technology.

3.1. Novel Nonaffine Nonlinear Approximation. The problem of controlling the plants characterized by models that are nonaffine in the control input vector is a difficult one. For the tracking control especially, the linearization may result in the design of sufficiently accurate controllers in the case of stabilization around the operating point; in the case of tracking of desired trajectories, the problem becomes much more difficult because the linearized model is time-varying. Hence, there is a clear need for the development of systematic control design techniques for nonlinear models that are nonaffine in $u$ and that are suitable for the case of tracking of desired trajectories.

For the nonaffine nonlinear model (4), the Taylor expansion of the nonlinear function $f[x(t), u(t)]$ with respect to $u(t)$ around $u(t-\tau)$ can result in

$$
\begin{gathered}
\dot{x}_{i}=x_{i+1}, \\
\dot{x}_{n}=f(x, u(t-\tau))+f_{d}(x, u(t-\tau)) \Delta u+R_{p}+d,
\end{gathered}
$$

where $\Delta u=u-u(t-\tau), f_{d}(x, u(t-\tau))=\partial f(x, u) /\left.\partial u\right|_{u=u(t-\tau)}$, and the remainder $R_{p}=f_{d d} \Delta u^{2} / 2$ is bounded by

$$
\left|R_{p}\right| \leq \frac{r_{p} \Delta u^{2}}{2},
$$

where $f_{d d}=\partial^{2} f(x, u) /\left.\partial^{2} u\right|_{u=\zeta}, \zeta$ is a point between $u$ and $u(t-\tau)$. Let $0 \leq\left|f_{d d}\right| \leq r_{p}$ with $r_{p}$ be a finite positive number.
The parameter $\tau>0$ is the updating input. It may be chosen as the sampling time in a sampled-data control system or as an integer multiple of the sampling time. A better choice of the parameter $\tau$ is the sampling because a larger $\tau$ may lead to an inaccurate approximation when the system function $f(x, u)$ varies quickly.

It is easy that (7) can be representation as the following form:

$$
\begin{gathered}
\dot{x}_{i}=x_{i+1}, \\
\dot{x}_{n}=f_{n}(x, u(t-\tau))+f_{d}(x, u(t-\tau)) u+d_{\xi}(t),
\end{gathered}
$$

where $d_{\xi}(t)=R_{p}+d(t)$ and

$$
f_{n}(x, u(t-\tau))=f(x, u(t-\tau))-f_{d}(x, u(t-\tau)) u(t-\tau) .
$$

In this paper, we assume that $\left|d_{\xi}(t)\right| \leq d_{M}$. To approximation accuracy, control input must satisfy the following assumption.

Assumption 1. Consider that $|\Delta u| \in[0, \delta]$ and $0<|\partial f / \partial u| \leq$ $\beta ; \delta$ and $\beta$ are two finite positive constants.

In Assumption $1,0<|\partial f / \partial u| \leq \beta$ means that the system (4) has a well-defined relative degree. $|\Delta u|$ should not be too large in order to limit the approximation error of the model (9) for a computed $u(t)$. In many actual process control systems and flight control systems, $|\Delta u(t)| \in[0, \delta]$ is a physical restriction of many practical systems because their states and outputs (actuators) cannot change too fast because of system "inertia”.

Convenient for the following statements, $u(t-\tau)$ is defined as $v(t)$, then (9) can be described as follows:

$$
\begin{gathered}
\dot{x}_{i}=x_{i+1}, \\
\dot{x}_{n}=f_{n}(x, v)+f_{d}(x, v) u+d_{\xi}(t) .
\end{gathered}
$$

Remark 1. By (9) and Assumption 1, it can be seen that $u(t-\tau)$ should be around the input $u$. If the time-delay $\tau$ is selected too large, the precision of approximation of simplified model will be reduced. So the selection of $\tau$ often requires experience. Theoretically, the smaller $\tau$ the better precision of global approximation, the best precision of global approximation if $\tau=0$. But $u$ is a control law to be solved, so it is unable to be realized. In order to obtain exact time-varying trim point, here, further improvement of the above-proposed method is given as follows. Considering lag property of the filtering as

$$
\dot{v}=-\lambda v+\lambda u
$$

then $\lim _{\lambda \rightarrow \infty} v=u$. This is a very good solution to the problem that $u(t-\tau)$ may be not around $u$. Here, $\lambda \rightarrow \infty$ is only a rigorous expression for mathematics meanings, in general, $\lambda \in[5,50]$. The filter (12) is not unique. The filtering $v$ can be completely replaced by an other filtering equation, such as higher-order differentiator. 
Remark 2. The traditional model simplification method is not global, mainly due to that the simplified model is fixed rather than time-varying model. It can be seen that (11) is a time-varying simplified model. The method which is proposed in this subsection can achieve the global approximation for nonlinear systems (4). So the proposed simplified model method can effectively solve the tracking control problem using affine nonlinear control strategy, such as sliding mode control, output-feedback control, and so on. backstepping control.

3.2. Adaptive Fuzzy-Neural Observer Design. The simplified model (11) can be described as

$$
\begin{gathered}
\dot{x}=A x+b\left[f_{n}(x, v)+f_{d}(x, v) u+d_{\xi}(t)\right], \\
y=c x .
\end{gathered}
$$

In order to ascertain subject for further elaboration, we shall use the following vectors, they are $\mathbb{x}=\left[x^{T}, v\right]^{T}$ and $\widehat{\mathbb{}}=$ $\left[\hat{x}^{T}, v\right]^{T}$.

We approximate the functions $f_{n}(\mathbb{x}), f_{d}(\mathbb{x})$ by using two fuzzy systems with their input being estimated by $\mathbb{x}$ as follows:

$$
f_{n}(\widehat{\mathbb{X}})=W_{f_{n}}^{T} P_{f_{n}}(\widehat{\mathbb{X}}), \quad f_{d}(\widehat{\mathbb{X}})=W_{f_{d}}^{T} P_{f_{d}}(\widehat{\mathbb{X}}) .
$$

The original functions $f_{n}(\mathbb{x})$ and $f_{d}(\mathbb{x})$ in $(24)$ can be expressed as

$$
\begin{aligned}
& f_{n}(\mathbb{x})=W_{f_{n}}^{* T} P_{f_{n}}(\mathbb{x})+\varepsilon_{f_{n}}, \\
& f_{d}(\mathbb{x})=W_{f_{d}}^{* T} P_{f_{d}}(\mathbb{x})+\varepsilon_{f_{d}},
\end{aligned}
$$

where $\varepsilon_{f_{n}}$ and $\varepsilon_{f_{d}}$ are the FNN functional reconstruction errors. In general, even given the best possible weight values, the given nonlinear function is not exactly approximated, and functional reconstruction error is remaining. $W_{f_{n}}^{*}$ and $W_{f_{d}}^{*}$ are the optimal parameter vectors required for analytical purpose satisfying

$$
\begin{aligned}
& W_{f_{n}}^{*}=\arg \min _{W_{f_{n}}}\left[\sup _{\mathbb{x} \in \Re^{n+1}}\left|f_{n}(\widehat{\mathbb{X}})-f_{n}(\mathbb{x})\right|\right], \\
& W_{f_{d}}^{*}=\arg \min _{W_{f_{d}}}\left[\sup _{\mathbb{x} \in \Re^{n+1}}\left|f_{d}(\widehat{\mathbb{X}})-f_{d}(\mathbb{x})\right|\right]
\end{aligned}
$$

bounded as $\left\|W_{f_{n}}^{*}\right\| \leq M_{f_{n}}$, $\left\|W_{f_{d}}^{*}\right\| \leq M_{f_{d}}$.

Using the FNN approximations, the dynamic equation of a fuzzy-neural observer that estimates the states in (13) is given as follows:

$$
\begin{gathered}
\dot{\hat{x}}=A \hat{x}+b_{0}\left[f_{n}(\widehat{\mathbb{X}})+f_{d}(\widehat{\mathbb{x}}) u\right]+L\left(y-c^{T} \hat{x}\right), \\
\hat{y}=c^{T} \widehat{x},
\end{gathered}
$$

where $L=\left[l_{1}, l_{2}, \ldots, l_{n}\right]^{T}$ is the observer gain vector, and $b_{0}$ will be designed later.
Defining the state and output estimate errors as $\tilde{x}=x-\widehat{x}$ and $\tilde{y}=y-\hat{y}$ yields the error dynamics from (13) and (17):

$$
\begin{aligned}
\dot{\tilde{x}}= & \bar{A} \widetilde{x}+b_{0}\left[\widetilde{W}_{f_{n}}^{T} P_{f_{n}}(\widehat{\mathbb{X}})+\widetilde{W}_{f_{d}}^{T} P_{f_{d}}(\widehat{\mathbb{X}}) u\right] \\
& +\left(b-b_{0}\right)\left[W_{f_{n}}^{* T} P_{f_{n}}(\mathbb{x})+W_{f_{d}}^{* T} P_{f_{d}}(\mathbb{x}) u\right] \\
& +b_{0}\left(d_{f_{n}}+d_{f_{d}} u\right)+b\left[d_{\xi}(t)+\varepsilon_{f_{n}}+\varepsilon_{f_{d}} u\right],
\end{aligned}
$$

where $\bar{A}=A-L c^{T}, \widetilde{W}_{f_{n}}=W_{f_{n}}^{*}-W_{f_{n}}, \widetilde{W}_{f_{d}}=W_{f_{d}}^{*}-W_{f_{d}}$, $d_{f_{n}}=W_{f_{n}}^{* T}\left[P_{f_{n}}(\mathbb{x})-P_{f_{n}}(\widehat{\mathbb{X}})\right]$, and $d_{f_{d}}=W_{f_{d}}^{* T}\left[P_{f_{d}}(\mathbb{x})-\right.$ $\left.P_{f_{d}}(\widehat{\mathbb{X}})\right]$. In general, the FNN basis function is bounded, for example, radial basis activation function, sigmoidal function, and spline function. This implies that every element of $P(\mathbb{x})-$ $P(\widehat{\mathbb{X}})$ is bounded, that is, $\left\|P_{f_{n}}(\mathbb{X})-P_{f_{n}}(\widehat{\mathbb{X}})\right\| \leq \widetilde{P}_{f_{n}}^{M}$ and $\| P_{f_{d}}(\mathbb{X})-$ $P_{f_{d}}(\widehat{\mathbb{X}}) \| \leq \widetilde{P}_{f_{d}}^{M}$ with $\widetilde{P}^{M}$ constant.

In order to construct the vector $b_{0}$, the algebraic Ricatilike equation $\bar{A}^{T} \Gamma+\Gamma \bar{A}+\Gamma^{2} \leq-Q$ for $Q>0$ is considered [20]. Using the positive definite matrix $\Gamma$, the vector $b_{0}$ is chosen as $b_{0}=\Gamma^{-1} c$. It is shown below that this choice will guarantee the stability of the observer.

Theorem 3. Consider the observer system (17). Let the update laws for the parameters of fuzzy-neural systems be

$$
\begin{gathered}
\dot{W}_{f_{n}}=\widetilde{y} \Gamma_{f_{n}} P_{f_{n}}(\widehat{x})-k_{f_{n}} W_{f_{n}}, \\
\dot{W}_{f_{d}}=\widetilde{y} \Gamma_{f_{d}} P_{f_{d}}(\widehat{x}) u-k_{f_{d}} W_{f_{d}},
\end{gathered}
$$

where $\Gamma_{f_{n}}=\Gamma_{f_{n}}^{T}>0, \Gamma_{f_{d}}=\Gamma_{f_{d}}^{T}>0$ and $k_{f_{n}}>0, k_{f_{d}}>0$. Then, the state estimation error and parameter estimation errors are uniformly ultimately bounded.

Proof. Consider the Lyapunov-function candidate

$$
V=\underbrace{\tilde{x}^{T} \Gamma \tilde{x}}_{V_{1}}+\underbrace{\widetilde{W}_{f_{n}}^{T} \Gamma_{f_{n}}^{-1} \widetilde{W}_{f_{n}}+\widetilde{W}_{f_{d}}^{T} \Gamma_{f_{d}}^{-1} \widetilde{W}_{f_{d}}}_{V_{2}} .
$$

The time derivative of $V_{1}$ is

$$
\begin{aligned}
& \dot{V}_{1}=\tilde{x}^{T}\left(\bar{A}^{T} \Gamma\right.+\Gamma \bar{A}) \tilde{x}+2 \widetilde{y}\left[\widetilde{W}_{f_{n}}^{T} P_{f_{n}}(\widehat{\mathbb{X}})+\widetilde{W}_{f_{d}}^{T} P_{f_{d}}(\widehat{\mathbb{x}}) u\right] \\
&+2 \widetilde{x}^{T} \Gamma\left\{\left(b-b_{0}\right)\left[W_{f_{n}}^{* T} P_{f_{n}}(\mathbb{x})+W_{f_{d}}^{* T} P_{f_{d}}(\mathbb{x}) u\right]\right. \\
&\left.+b_{0}\left(d_{f_{n}}+d_{f_{d}} u\right)+b\left[d_{\xi}(t)+\varepsilon_{f_{n}}+\varepsilon_{f_{d}} u\right]\right\} .
\end{aligned}
$$

Since the $P_{f_{n}}$ and $P_{f_{d}}$ are bounded, that is, $\left\|P_{f_{n}}(\mathbb{x})\right\| \leq P_{f_{n}}^{M}$ and $\left\|P_{f_{d}}(\mathbb{X})\right\| \leq P_{f_{d}}^{M}$ with $P_{f_{n}}^{M}$ and $P_{f_{d}}^{M}$ as constants, thus,

$$
\begin{aligned}
& \left(b-b_{0}\right)\left[W_{f_{n}}^{* T} P_{f_{n}}(\mathbb{x})+W_{f_{d}}^{* T} P_{f_{d}}(\mathbb{x}) u\right]+b_{0}\left(d_{f_{n}}+d_{f_{d}} u\right) \\
& +b\left[d_{\xi}(t)+\varepsilon_{f_{n}}+\varepsilon_{f_{d}} u\right] \leq \Upsilon
\end{aligned}
$$


with

$$
\begin{aligned}
\Upsilon= & \left\|\left(b-b_{0}\right)\right\|\left[M_{f_{n}} P_{f_{n}}^{M}+M_{f_{d}} P_{f_{d}}^{M} \bar{u}\right] \\
& +\left\|b_{0}\right\|\left(M_{f_{n}} \widetilde{P}_{f_{n}}^{M}+M_{f_{d}} \widetilde{P}_{f_{d}}^{M} \bar{u}\right) \\
& +b\left[d_{M}+\varepsilon_{f_{n} M}+\varepsilon_{f_{d} M} \bar{u}\right] .
\end{aligned}
$$

By using $2 a_{1}^{T} b_{1} \leq a_{1}^{T} a_{1}+b_{1}^{T} b_{1}$, it can be shown that

$$
\begin{aligned}
& 2 \tilde{x}^{T} \Gamma\left\{\left(b-b_{0}\right)\left[W_{f_{n}}^{* T} P_{f_{n}}(\mathbb{x})+W_{f_{d}}^{* T} P_{f_{d}}(\mathbb{x}) u\right]+b_{0}\left(d_{f_{n}}+d_{f_{d}} u\right)\right. \\
& \left.\quad+b\left[d_{\xi}(t)+\varepsilon_{f_{n}}+\varepsilon_{f_{d}} u\right]\right\} \\
& \leq \tilde{x}^{T} \Gamma^{2} \tilde{x}+\Upsilon^{2} .
\end{aligned}
$$

Substituting (24) into (21) yields

$$
\dot{V}_{1} \leq-\tilde{x}^{T} Q \tilde{x}+2 \widetilde{y}\left[\widetilde{W}_{f_{n}}^{T} P_{f_{n}}(\widehat{\mathbb{x}})+\widetilde{W}_{f_{d}}^{T} P_{f_{d}}(\widehat{\mathbb{x}}) u\right]+\Upsilon^{2}
$$

Then, $\dot{V}$ follows as

$$
\dot{V} \leq-\tilde{x}^{T} Q \tilde{x}+2 k_{f_{n}} \widetilde{W}_{f_{n}}^{T} W_{f_{n}}+2 k_{f_{g}} \widetilde{W}_{f_{g}}^{T} W_{f_{g}}+\Upsilon^{2}
$$

where the update laws (19) have been used. By completion of squares, it follows that

$$
\begin{aligned}
\dot{V} \leq-\lambda_{\min }(Q)\left\{\|\tilde{x}\|^{2}+\frac{2 k_{f_{n}}}{\lambda_{\min }(Q)}\left(\left\|\widetilde{W}_{f_{n}}^{T}\right\|-\frac{1}{2}\left\|W_{f_{n}}^{*}\right\|\right)^{2}\right. \\
+\frac{2 k_{f_{d}}}{\lambda_{\min }(Q)}\left(\left\|\widetilde{W}_{f_{d}}^{T}\right\|-\frac{1}{2}\left\|W_{f_{d}}^{*}\right\|\right)^{2} \\
-\left[\frac{k_{f_{n}}}{2 \lambda_{\min }(Q)}\left\|W_{f_{n}}^{*}\right\|^{2}+\frac{k_{f_{d}}}{2 \lambda_{\min }(Q)}\left\|W_{f_{d}}^{*}\right\|^{2}\right. \\
\left.\left.+\frac{1}{\lambda_{\min }(Q)} \Upsilon^{2}\right]\right\},
\end{aligned}
$$

which is guaranteed negative as long as either

$$
\|\tilde{x}\|>\sqrt{\frac{k_{f_{n}}\left\|W_{f_{n}}^{*}\right\|^{2}}{2 \lambda_{\min }(Q)}+\frac{k_{f_{d}}\left\|W_{f_{d}}^{*}\right\|^{2}}{2 \lambda_{\min }(Q)}+\frac{\Upsilon^{2}}{\lambda_{\min }(Q)}}=B_{x}
$$

or

$$
\begin{aligned}
& \left\|\widetilde{W}_{f_{n}}^{T}\right\|>\sqrt{\frac{\left\|W_{f_{n}}^{*}\right\|^{2}}{4}+\frac{\left\|W_{f_{d}}^{*}\right\|^{2}}{4}+\frac{\Upsilon^{2}}{2}}+\frac{\left\|W_{f_{n}}^{*}\right\|}{2}=B_{f_{n}}, \\
& \left\|\widetilde{W}_{f_{d}}^{T}\right\|>\sqrt{\frac{\left\|W_{f_{n}}^{*}\right\|^{2}}{4}+\frac{\left\|W_{f_{d}}^{*}\right\|^{2}}{4}+\frac{\Upsilon^{2}}{2}}+\frac{\left\|W_{f_{d}}^{*}\right\|}{2}=B_{f_{d}} .
\end{aligned}
$$

Defining a vector $\mathbb{Z}=\left[\tilde{x}^{T}, \widetilde{W}_{f_{n}}^{T}, \widetilde{W}_{f_{d}}^{T}\right]^{T}$, thus, $\dot{V}$ is negative outside the compact set: $M_{\sigma}=\left\{\mathbb{Z} \in M_{z} \mid\|\mathbb{z}\| \leq \sigma\right\}$, where $\sigma=\max \left(B_{x}, B_{f_{n}}, B_{f_{d}}\right)$. We can guarantee that $\alpha<\beta$ which can be made by increasing $\lambda_{\min }(Q)$, and therefore ultimate boundedness of $\mathbb{Z}$, where $\alpha=\max _{\|\mathbb{Z}\|=\sigma} V$ and $\beta=\min _{\|\mathbb{Z}\|=R} V$.

3.3. Controller Design and Stability Analysis. Define tracking error as

$$
E=x-x_{d}=\left[e, \dot{e}, \ldots, e^{(n-1)}\right]^{T}
$$

where $e=x_{1}-x_{1 d}$. Define the following terminal sliding mode surface:

$$
\sigma(x, t)=C E-W(t)
$$

where $C=\left[c_{1}, c_{2}, \ldots, c_{n}\right], c_{i}(i=1, \ldots, n)$ is positive constant, and $c_{n}=1$. And $W(t)=C P(t)$, where $P(t)=$ $\left[p(t), \dot{p}(t), \ldots, p^{(n-1)}(t)\right]^{T}$.

Assumption 2. Consider terminal function $p(t): \mathscr{R}_{+} \rightarrow \mathscr{R}$, $p(t) \in C^{n}[0, \infty), p, \dot{p}, \ldots, p^{(n)} \in L^{\infty}, p(t)$ is finite in interval $[0, T], E(0)=P(0), \dot{E}(0)=\dot{P}(0)$, that is, $p(0)=e(0), \dot{p}(0)=$ $\dot{e}(0), \ldots, p^{(n)}(0)=e^{(n)}(0)$. Moreover, $p=0, \dot{p}=0, \cdots, p^{(n)}=$ 0 , for $t \geq T \cdot C^{n}[0, \infty)$ represents the set of all $n$ rank differentiable continuous functions defined in $[0, \infty)$.

Define terminal function $p(t)$ for $(4)$ as

$$
p(t)= \begin{cases}\sum_{j=0}^{n}\left(\sum_{l=0}^{n} \frac{a_{j l}}{T^{j-l+n+1}} e_{i}(0)^{(l)}\right) t^{j+n+1} & \\ \quad+\sum_{k=0}^{n} \frac{1}{k !} e_{i}(0)^{(k)} t^{k} & t \leq T \\ 0 & t>T,\end{cases}
$$

where $a_{j l}$ can be defined by using Assumption 3 .

Theorem 4. Suppose that the control law is

$$
\begin{gathered}
u(t)=-\frac{1}{b_{0} f_{d}(\widehat{\mathbb{X}})}\left\{b_{0} f_{n}(\widehat{\mathbb{X}})+l_{n} \tilde{y}-x_{1 d}^{(n)}-p^{(n)}(t)\right. \\
\left.\quad+c_{n}^{-1} \sum_{k=1}^{n-1}\left[\widehat{e}^{(k)}-p^{(k)}(t)\right]\right\} \\
-\frac{1}{b_{0} f_{d}(\widehat{\mathbb{X}})} \frac{c_{n} \sigma(x, t)}{\left\|c_{n} \sigma(x, t)\right\|}\{D(t)+K\},
\end{gathered}
$$

where $K>0$, then $\sigma(x, t)$ will reach zero in finite time $T$. Furthermore, the sates $x$ will converge to zero in finite time $T$, and $D$ is a positive constant value designed as below.

Proof. Consider a Lyapunov-function candidate as follows:

$$
V_{3}(t)=\frac{1}{2} \sigma^{T} \sigma
$$


The derivative of $\sigma(x, t)$ is

$$
\begin{aligned}
\dot{\sigma}(x, t)= & C \dot{E}-C \dot{P}(t) \\
= & C \cdot\left[\dot{e}^{T} \cdots e^{(n-1) T}\right]^{T}-C \cdot\left[\dot{p}(t)^{T} \cdots p^{(n-1)}(t)^{T}\right]^{T} \\
= & c_{n}\left[x_{1}^{(n)}-x_{1 d}^{(n)}-p^{(n)}(t)\right]+\sum_{k=1}^{n-1} c_{k}\left[e^{(k)}-p^{(k)}(t)\right] \\
= & c_{n}\left[\widehat{x}_{1}^{(n)}-x_{1 d}^{(n)}-p^{(n)}(t)\right]+\sum_{k=1}^{n-1} c_{k}\left[\hat{e}^{(k)}-p^{(k)}(t)\right] \\
& +\sum_{k=2}^{n} c_{k} \widetilde{x}_{k} \\
= & c_{n}\left[b_{0} f_{n}(\widehat{\mathbb{x}})+b_{0} f_{d}(\widehat{\mathbb{x}}) u+l_{n} \widetilde{y}^{(n)} x_{1 d}^{(n)}-p^{(n)}(t)\right] \\
& +\sum_{k=1}^{n-1} c_{k}\left[\widehat{e}^{(k)}-p^{(k)}(t)\right]+\sum_{k=2}^{n} c_{k} \tilde{x}_{k},
\end{aligned}
$$

where $\widehat{e}=\widehat{x}-x_{d}, \dot{\hat{e}}=\widehat{x}_{2}-\dot{x}_{d}, \ldots, \widehat{e}^{(n-1)}=\widehat{x}_{n}=\widehat{x}_{d}^{(n-1)}$. Then

$$
\begin{aligned}
\dot{V}_{3}(t)= & \sigma^{T} \dot{\sigma} \\
= & \sigma^{T} c_{n}\left\{b_{0} f_{n}(\widehat{\mathbb{x}})+l_{n} \widetilde{y}-x_{1 d}^{(n)}-p^{(n)}(t)\right. \\
& \left.+c_{n}^{-1} \sum_{k=1}^{n-1}\left[\widehat{e}^{(k)}-p^{(k)}(t)\right]\right\}+\sigma^{T} c_{n} b_{0} f_{d}(\widehat{\mathbb{x}}) u \\
& +\sigma^{T} c_{n} c_{n}^{-1} \sum_{k=2}^{n} c_{k} \widetilde{x}_{k} \\
\leq & \sigma^{T} c_{n}\left\{b_{0} f_{n}(\widehat{\mathbb{x}})+l_{n} \widetilde{y}-x_{1 d}^{(n)}-p^{(n)}(t)\right. \\
& \left.+c_{n}^{-1} \sum_{k=1}^{n-1}\left[\hat{e}^{(k)}-p^{(k)}(t)\right]\right\}+\sigma^{T} c_{n} b_{0} f_{d}(\widehat{\mathbb{x}}) u \\
& +\left\|\sigma^{T} c_{n}\right\|\left\|c_{n}^{-1} \sum_{k=2}^{n} c_{k} \widetilde{x}_{k}\right\| .
\end{aligned}
$$

By Theorem 3, it is easily get that $\left|c_{n}^{-1} \sum_{k=2}^{n} c_{k} \tilde{x}_{k}\right| \leq D(t)$.

Substituting the control law (34) into $\dot{V}_{3}(t)$ yields

$$
\dot{V}_{3}(t) \leq-\frac{\sigma^{T} c_{n} c_{n}^{T} \sigma}{\left\|c_{n} \sigma\right\|}\{D(t)+K\}
$$

because of

$$
\sigma^{T} c_{n} c_{n}^{T} \sigma=\left\|c_{n} \sigma\right\|^{2}
$$

so

$$
\dot{V}_{3}(t) \leq 0 \text {. }
$$

If $|\sigma| \neq 0$, then $\dot{V}_{3}(t)<0$. This means that this Lyapunov function will decrease gradually, and the sliding surface $\sigma(x, t)$ will converge to zero. The proof is completed.

$$
\begin{aligned}
& \text { Remark 5. It follows from Assumption } 2 \text { that } \\
& \qquad \sigma(x, 0)=C E(0)-W(0)=C[E(0)-P(0)]=0 .
\end{aligned}
$$

According to Lyapunov analysis, $\sigma(x, t)=0$ can be achieved all the time. This indicates that the reaching phase in terminal sliding mode control can be eliminated, and global robustness can be guaranteed.

Remark 6. In order to reduce chattering which is caused by discontinuous control signal, a continuous function vector is used to the controller design. In control law (34), $c_{n}^{T} \sigma(x, t) /\left\|c_{n} \sigma(x, t)\right\|$ is replaced by the continuous function vector $S_{\delta}$ defined by

$$
S_{\delta}=\frac{c_{n}^{T} \sigma(x, t)}{\left\|c_{n} \sigma(x, t)\right\|+\delta}
$$

with $\delta=\delta_{0}+\delta_{1}\|e\|$, where $\delta_{0}, \delta_{1}$ are two positive constants.

\section{Simulation Results}

This section presents the simulation results of the proposed tracking controller to illustrate that the stability of the closed-loop system is guaranteed, and all signal involved are bounded. Consider the nonlinear system

$$
\begin{gathered}
\dot{x}_{1}=x_{2}, \\
\dot{x}_{2}=0.2\left(1+e^{x_{1} x_{2}}\right)\left(2+\sin \left(x_{2}\right)\right)\left(u+e^{u}-1\right)+d(t), \\
y=x_{1},
\end{gathered}
$$

where external disturbance $d(t)=5 \sin (7 \pi t)$. Define terminal function $p(t)$ for dynamics of (43) as

$$
p(t)= \begin{cases}\sum_{j=0}^{2}\left(\sum_{l=0}^{2} \frac{a_{j l}}{T^{j-l+3}} e_{i}(0)^{(l)}\right) t^{j+3} & \\ +\sum_{k=0}^{2} \frac{1}{k !} e_{i}(0)^{k} t^{k} & t \leq T \\ 0 & t>T\end{cases}
$$

according to Assumption 3; function $p(t), \dot{p}(t)$, and $\ddot{p}(t)$ can be equal to zero at time $t=T$ by designing $a_{j l}(j=0,1,2, l=$ $0,1,2)$. That is, if we design $a_{j l}$ according to the following 
equations, $p(t), \dot{p}(t)$, and $\ddot{p}(t)$ can all be equal to zero at time $t=T$ :

$$
\begin{gathered}
a_{00}+a_{10}+a_{20}=-1, \\
3 a_{00}+4 a_{10}+5 a_{20}=0, \\
6 a_{00}+12 a_{10}+20 a_{20}=0, \\
a_{01}+a_{11}+a_{21}=-1, \\
3 a_{01}+4 a_{11}+5 a_{21}=-1, \\
6 a_{01}+12 a_{11}+20 a_{21}=0, \\
a_{02}+a_{12}+a_{22}=-\frac{1}{2}, \\
3 a_{02}+4 a_{12}+5 a_{22}=-1, \\
6 a_{02}+12 a_{12}+20 a_{22}=-1 .
\end{gathered}
$$

Based on the three groups of (45), parameters $a_{j l}$ can be solved as

$$
\begin{array}{rrr}
a_{00}=-10, & a_{01}=-6, & a_{02}=-1.5, \\
a_{10}=15, & a_{11}=8, & a_{12}=1.5, \\
a_{20}=-6, & a_{21}=-3, & a_{22}=-0.5 .
\end{array}
$$

From (44)-(46), $p(t)$ can be written as

$$
p(t)= \begin{cases}e_{0}+\dot{e}_{0} t+\frac{1}{2} \ddot{e}_{0} t^{2}+\left(\frac{a_{00}}{T^{3}} e_{0}+\frac{a_{01}}{T^{2}} \dot{e}_{0}+\frac{a_{02}}{T} \ddot{e}_{0}\right) t^{3} & \\ +\left(\frac{a_{10}}{T^{4}} e_{0}+\frac{a_{11}}{T^{3}} \dot{e}_{0}+\frac{a_{12}}{T^{2}} \ddot{e}_{0}\right) t^{4} & \\ +\left(\frac{a_{20}}{T^{5}} e_{0}+\frac{a_{21}}{T^{4}} \dot{e}_{0}+\frac{a_{22}}{T^{3}} \ddot{e}_{0}\right) t^{5}, & t \leq T \\ 0, & t>T .\end{cases}
$$

We choose control parameters as $c_{2}=50, c_{1}=1$, and $K=0$, and terminal time is chosen as $T=1.0$. The control law is now chosen as

$$
\begin{aligned}
u(t)= & -\frac{1}{b_{0} f_{d}(\widehat{\mathbb{x}})}\left\{b_{0} f_{n}(\widehat{\mathbb{X}})+l_{n} \tilde{y}-\ddot{x}_{1 d}-\ddot{p}(t)+c_{2}^{-1} \dot{\hat{e}}-c_{2}^{-1} \dot{p}(t)\right\} \\
& -\frac{1}{b_{0} f_{d}(\widehat{\mathbb{x}})} \frac{c_{2} \sigma(x, t)}{\left\|c_{2} \sigma(x, t)\right\|}\{D(t)+K\} .
\end{aligned}
$$

Then the observer for the system (43) is given by (17). We can apply Theorem 3 with the following simulation parameters: observer gain $L=[1000,8000]$, weight-tuning parameters $\Gamma_{f_{n}}=\operatorname{diag}\left[5 \times 10^{4}\right]$, and $\Gamma_{f_{d}}=\operatorname{diag}\left[5 \times 10^{3}\right]$. And $k_{f_{n}}=k_{f_{d}}=$ 0.001 .

The desired output is $x_{1 d}=\sin t$. The initial conditions are $x(0)=[0,0.5]^{T} . \widehat{x}(0)=[0.1,0,0]^{T}$. The response of the system (43) and adaptive fuzzy-neural observer with the terminal SMC law is shown in Figure 2. From Figure 2, we can
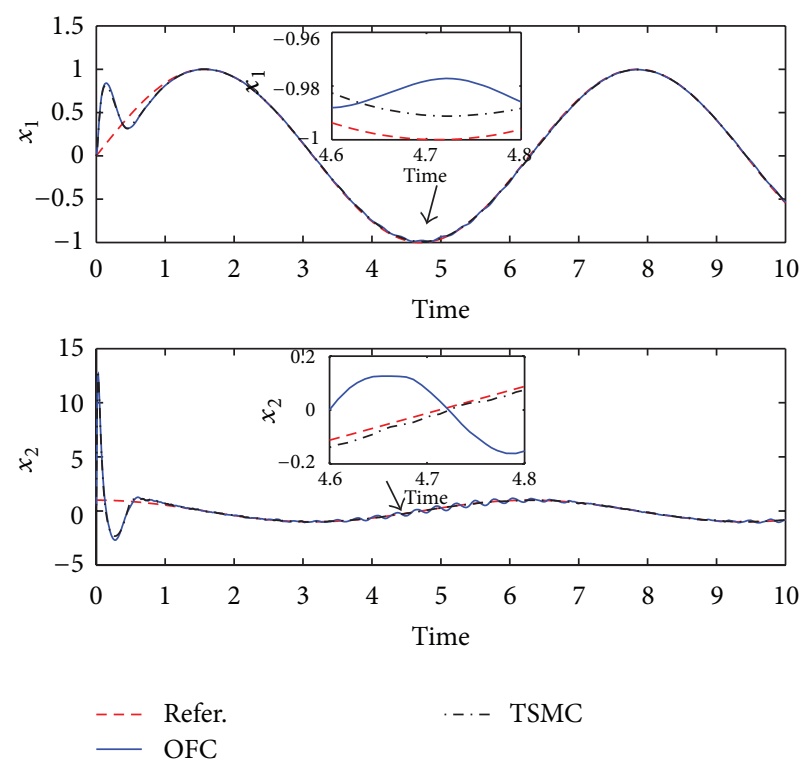

FIGURE 2: System response with the proposed tracking controller.
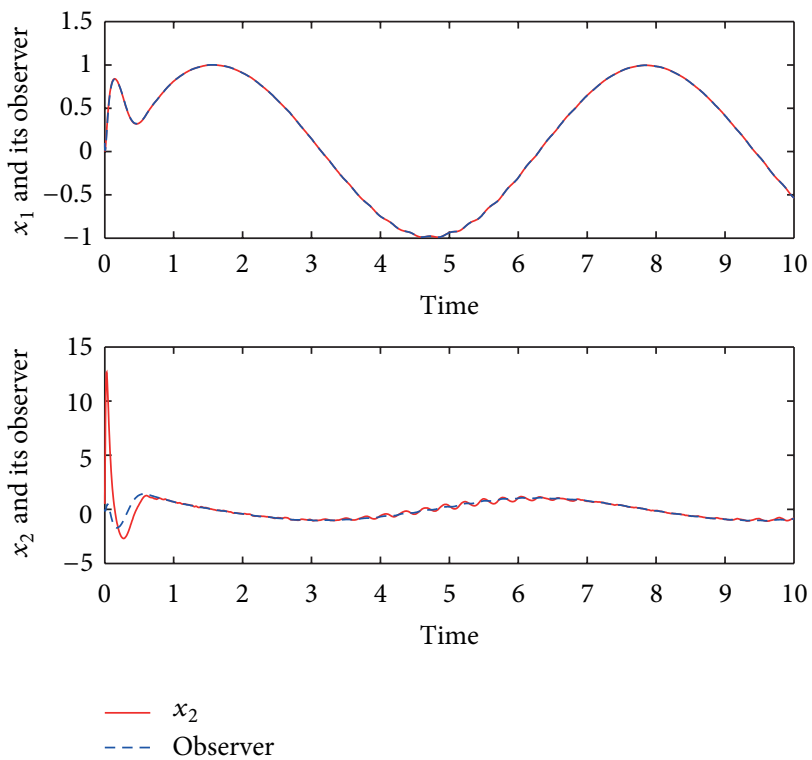

FIGURE 3: System states and observer values.

obtain a better tracking ability by terminal SMC than output feedback control (OFC) method of $[21,22]$. Figure 3 shows the states and observer values, and Figure 4 shows the errors of observer.

\section{Conclusion}

We have carried out a systematic study on fuzzy-neural observer-based terminal SMC in this paper, based on an adaptive fuzzy-neural observer which is used to identify the model and estimate the states. So the information of mathematical model and states does not require to know, 

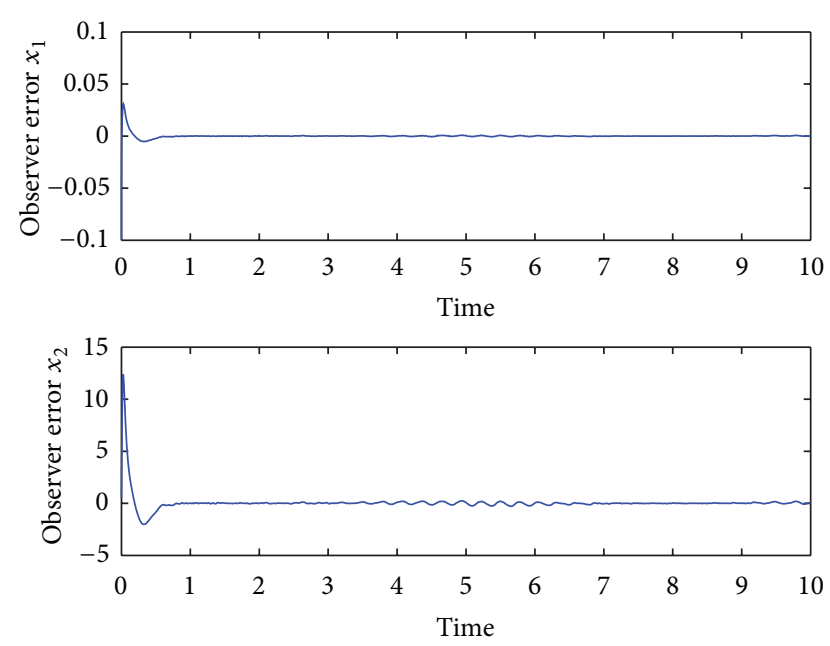

Figure 4: Observer error of states.

only using the measurable output. The proposed nonlinear tracking control scheme can guarantee the asymptotic output tracking of the closed-loop control systems in spite of unknown uncertainties/disturbances. Finally, simulation results are provided on a nonaffine nonlinear system to show the effective and advantages of the new control strategy.

\section{Acknowledgments}

This work was partially supported by the National Natural Science Foundation of China (61273171, 61034005, and 61174058), the National Aerospace Science Foundation of China (2011ZA52009, 2012ZA52017), PAPD, Funding of Jiangsu Innovation Program for Graduate Education (CXZZ11-0213), and funding for Outstanding Doctoral Dissertation in NUAA (BCXJ12-04).

\section{References}

[1] P. Shi, Y. Xia, G. P. Liu, and D. Rees, "On designing of slidingmode control for stochastic jump systems," IEEE Transactions on Automatic Control, vol. 51, no. 1, pp. 97-103, 2006.

[2] J. Zhang, P. Shi, and Y. Xia, "Robust adaptive sliding-mode control for fuzzy systems with mismatched uncertainties," IEEE Transactions on Fuzzy Systems, vol. 18, no. 4, pp. 700-711, 2010.

[3] L. Wu, P. Shi, and H. Gao, "State estimation and sliding-mode control of Markovian jump singular systems," IEEE Transactions on Automatic Control, vol. 55, no. 5, pp. 1213-1219, 2010.

[4] Q. Khan, A. I. Bhatti, M. Iqbal, and Q. Ahmed, "Dynamic integral sliding mode control for SISO uncertain nonlinear systems," International Journal of Innovative Computing, Information and Control, vol. 8, no. 7, pp. 4621-4633, 2012.

[5] L. Wu and D. W. C. Ho, "Sliding mode control of singular stochastic hybrid systems," Automatica, vol. 46, no. 4, pp. 779783, 2010.

[6] J. Fei and M. Xin, "Robust adaptive sliding mode controller for semi-active vehicle suspension system," International Journal of Innovative Computing, Information and Control, vol. 8, no. 1, pp. 691-700, 2012.
[7] L. Wu and W. X. Zheng, "Passivity-based sliding mode control of uncertain singular time-delay systems," Automatica, vol. 45, no. 9, pp. 2120-2127, 2009.

[8] Y. Wu, X. Yu, and Z. Man, "Terminal sliding mode control design for uncertain dynamic systems," Systems \& Control Letters, vol. 34, no. 5, pp. 281-287, 1998.

[9] J. Wang, L. Zou, H. Peng, and G. Zhang, "An extended spiking neural P system for fuzzy knowledge representation," International Journal of Innovative Computing, Information and Control, vol. 7, no. 7, pp. 3709-3724, 2011.

[10] W. Pawlus, H. R. Karimi, and K. G. Robbersmyr, "Data-based modeling of vehicle collisions by nonlinear autoregressive model and feedforward neural network," Information Sciences, 2012.

[11] D. Jia and B. You, "Study on novel plasma arc cutting technology based on PIDNN-FUZZY controller," International Journal of Innovative Computing, Information and Control, vol. 7, no. 7, pp. 4171-4182, 2011.

[12] L. Wu, X. Su, P. Shi, and J. Qiu, "Model approximation for discrete-time state-delay systems in the T-S fuzzy framework," IEEE Transactions on Fuzzy Systems, vol. 19, no. 2, pp. 366-378, 2011.

[13] T.-C. Lin, S.-W. Chang, and C.-H. Hsu, "Robust adaptive fuzzy sliding mode control for a class of uncertain discretetime nonlinear systems," International Journal of Innovative Computing, Information and Control, vol. 8, no. 1, pp. 347-359, 2012.

[14] X. Su, P. Shi, L. Wu, and Y.-D. Song, "A novel approach to filter design for T-S fuzzy discrete-time systems with time-varying delay," IEEE Transactions on Fuzzy Systems, vol. 20, no. 6, Article ID 6189779, pp. 1114-1129, 2012.

[15] C. M. Lin and C. F. Hsu, "Supervisory recurrent fuzzy neural network control of wing rock for slender delta wings," IEEE Transactions on Fuzzy Systems, vol. 12, no. 5, pp. 733-742, 2004.

[16] C. M. Lin and C. F. Hsu, "Supervisory recurrent fuzzy neural network control of wing rock for slender delta wings," IEEE Transactions on Fuzzy Systems, vol. 12, no. 5, pp. 733-742, 2004.

[17] B. Jiang, Z. Gao, P. Shi, and Y. Xu, "Adaptive fault-tolerant tracking control of near-space vehicle using TakagiSugeno fuzzy models," IEEE Transactions on Fuzzy Systems, vol. 18, no. 5, pp. 1000-1007, 2010.

[18] S. J. Lopez, O. C. Nieto, and J. I. C. Oria, "Non-parametric modeling of uncertain hyperbolic partial differential equations using pseudo-high order sliding mode observers," International Journal of Innovative Computing, Information and Control, vol. 8, no. 3, pp. 1501-1521, 2012.

[19] Y. H. Kim, L. Frank, and C. T. Abdallah, "A dynamic recurrent neural-network-based adaptive observer for a class of nonlinear systems," Automatica, vol. 33, no. 8, pp. 1539-1543, 1997.

[20] M.-L. Ni and M. J. Er, "Stability of linear systems with delayed perturbations: an LMI approach," IEEE Transactions on Circuits and Systems. I, vol. 49, no. 1, pp. 108-112, 2002.

[21] S. K. Nguang and P. Shi, " $H_{\infty}$ fuzzy output feedback control design for nonlinear systems: an LMI approach," IEEE Transactions on Fuzzy Systems, vol. 11, no. 3, pp. 331-340, 2003.

[22] Y. Wang, T. Chai, and Y. Zhang, "State observer-based adaptive fuzzy output-feedback control for a class of uncertain nonlinear systems," Information Sciences, vol. 180, no. 24, pp. 5029-5040, 2010. 


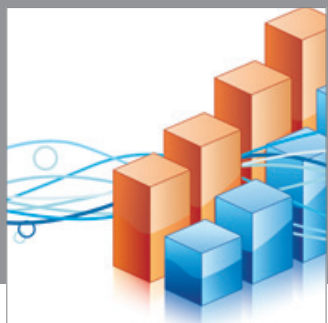

Advances in

Operations Research

mansans

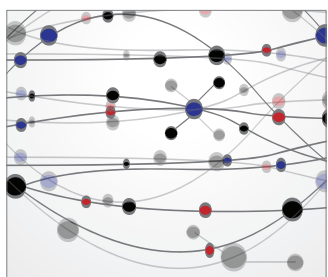

The Scientific World Journal
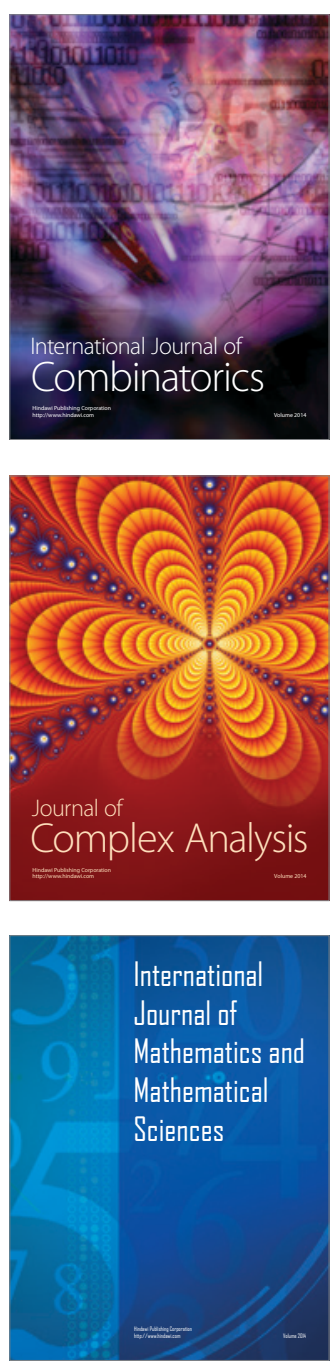
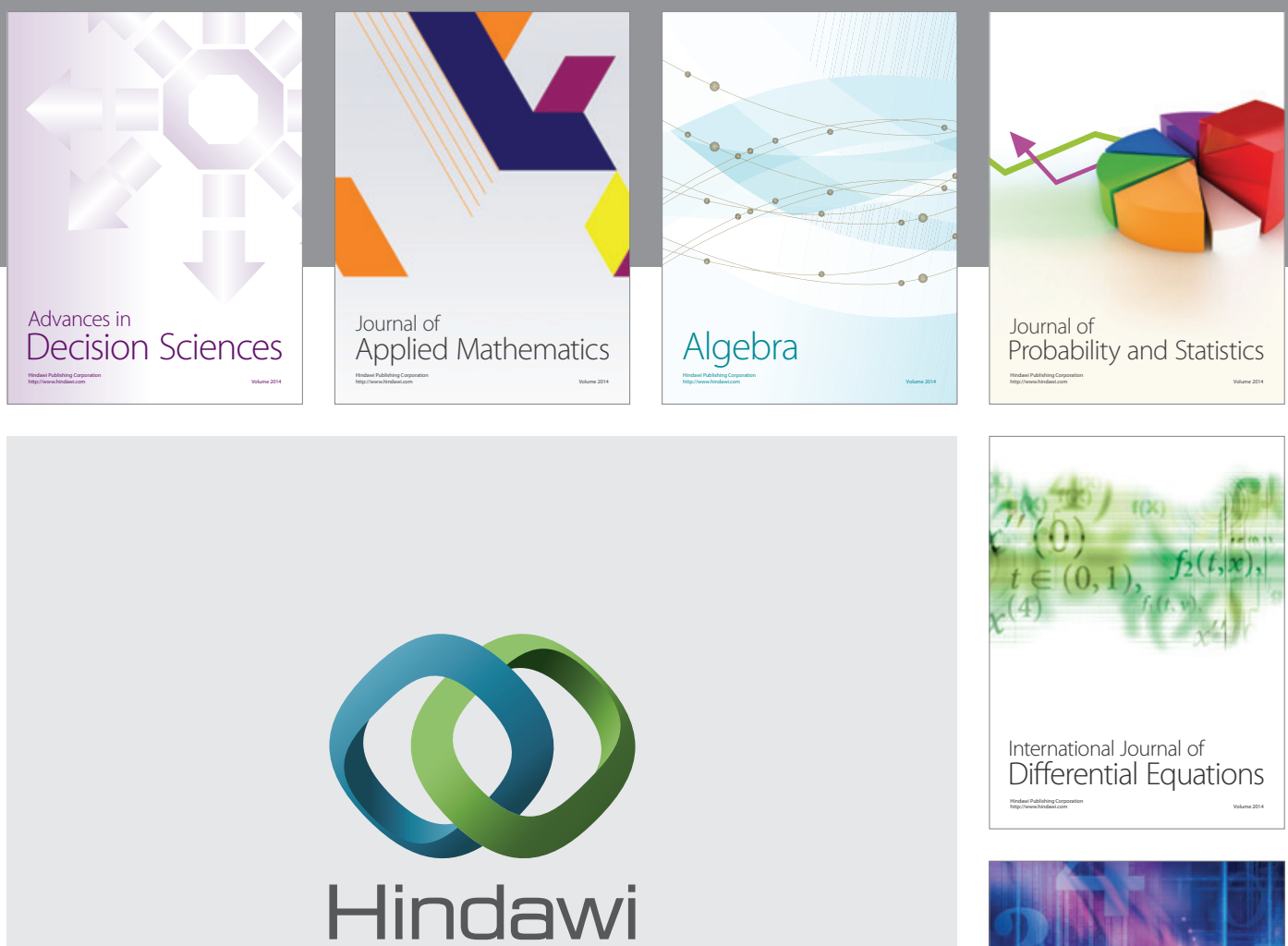

Submit your manuscripts at http://www.hindawi.com
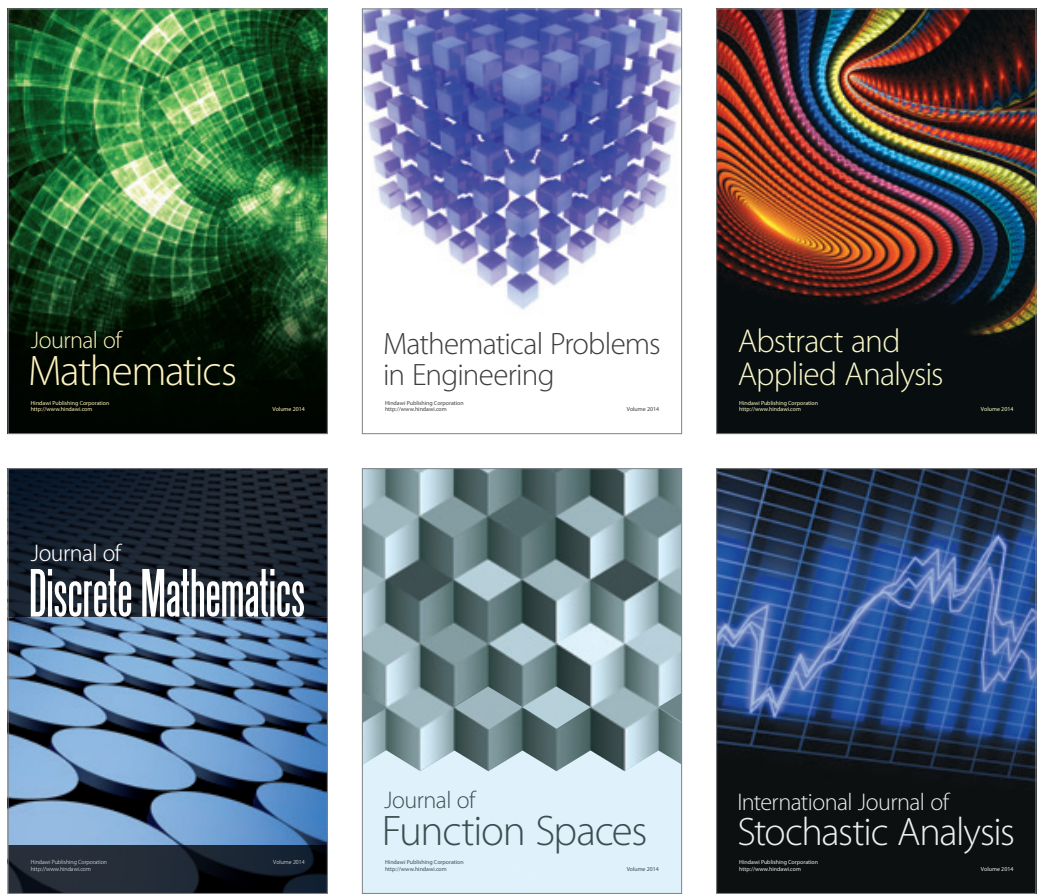

Journal of

Function Spaces

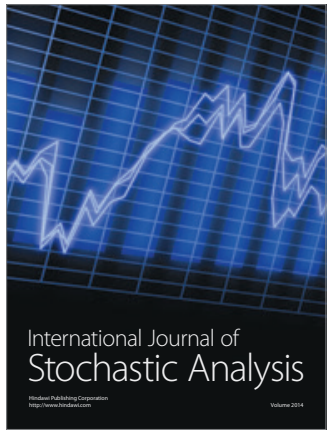

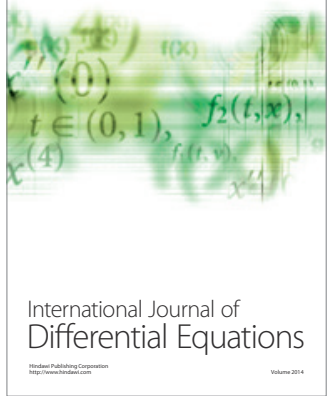
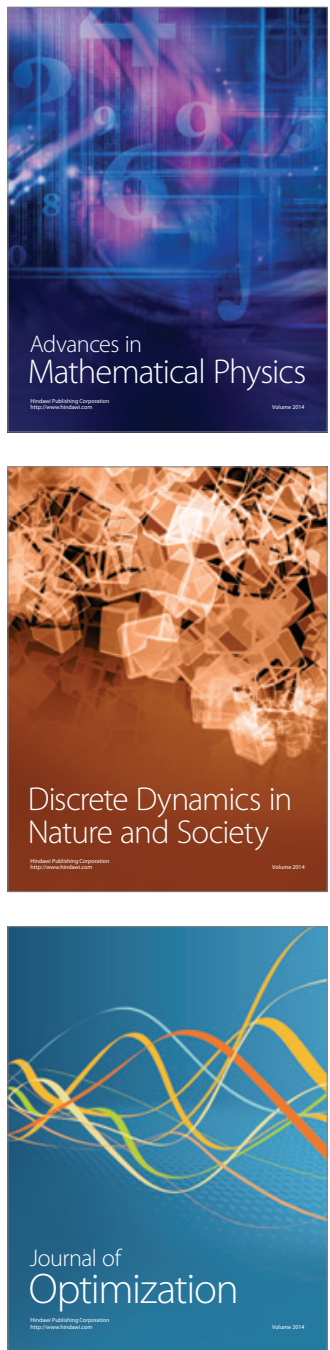